\title{
¿CONTRIBUYEN LOS ENSAYOS CLÍNICOS AL DESARROLLO DE LA INVESTIGACIÓN EN EL PERÚ?, ¿CÓMO LOGRARLO?
}

\section{DO CLINICAL TRIALS CONTRIBUTE TO RESEARCH DEVELOPMENT IN PERU? HOW CAN THAT BE ACHIEVED?}

\author{
Germán Málaga ${ }^{1,2, a}$ Ana Zúñiga-Rivera 2,3,b
}

\author{
CRONICAS Centro de Excelencia en enfermedades crónicas, Universidad Peruana Cayetano Heredia. Lima, Perú \\ Unidad de Conocimiento y Evidencia, Universidad Peruana Cayetano Heredia. Lima, Perú. \\ Clínica Montesur. Lima, Perú. \\ Médico internista magister en Medicina; ${ }^{b}$ médico pediatra neonatóloga \\ Recibido: 04-11-12; Aprobado: 14-11-12
}

\begin{abstract}
RESUMEN
La participación del Instituto Nacional de Salud como ente rector en la evaluación ética, metodológica y, por consiguiente, a cargo de la aprobación de la ejecución de estudios, data del año 1995. Desde entonces ha existido un gran incremento de ensayos clínicos hasta el 2009, por lo que se realizó un análisis preliminar para evaluar si este incremento ha repercutido en la producción de ensayos clínicos sobre tópicos endémicos o problemas de carga de enfermedad que deben ser investigados y resueltos por los peruanos. Para ello, se realizó una búsqueda en SCOPUS Y MEDLINE, pudiendo identificar 102 ensayos clínicos en humanos, diseñados, ejecutados y realizados en el Perú sobre problemas endémicos o propios del Perú. La apreciación en el tiempo no permite afirmar que haya ocurrido un incremento en paralelo, ni que se hayan incluido a nuevos grupos de investigación o nuevos investigadores, no se investiga sobre problemas endémicos de nuestro país, no se ha realizado por ejemplo ningún ensayo clínico sobre la enfermedad de Carrión, son muy pocos los ensayos clínicos promovidos durante este tiempo por instituciones académicas y de la sociedad civil. Teniendo en cuenta ello, podemos afirmar que no se aprecia una influencia positiva en la generación de ensayos clínicos autóctonos, sobre problemas locales que a los peruanos les corresponde resolver.
\end{abstract}

Palabras clave: Ensayos clínicos aleatorios; Industria farmacéutica; Regulación y fiscalización en salud; Indicadores de producción científica (fuente: DeCS BIREME).

\begin{abstract}
The participation of the National Institute of Health as a leader in ethical and methodological assessment and its responsibility to approve the implementation of studies dates back to 1995. Since then, clinical trials have increased significantly until 2009; for this reason, a preliminary analysis was made to assess whether this increase has had an impact in the production of clinical trials on endemic topics or disease burden problems that must be researched and resolved by Peruvian people. For this purpose, a search in SCOPUS and MEDLINE was conducted, with 102 clinical trials in humans found, all of them designed, implemented and carried out in Peru on endemic or domestic health issues. Observation over time does not reveal any parallel increase or the inclusion of new research groups or researchers, no research on endemic problems has been carried out in our country; for example, clinical trials on the "Carrion's Disease" have not been conducted. Very few clinical trials have been promoted during this period by academic institutions and the civil society. Taking this into consideration, we can affirm that there is no positive influence in the generation of native clinical trials on local problems that Peruvian people need to resolve.
\end{abstract}

Key words: Clinical trials, randomized; Drug industry; Health care coordination and monitoring; Scientific publication indicators (source: MeSH NLM).

Citar como: Málaga G, Zúñiga-Rivera A. ¿Contribuyen los ensayos clínicos al desarrollo de la investigación en el Perú?, ¿ cómo lograrlo? Rev Peru Med Exp Salud Publica. 2012;29(4):529-34. 


\section{INTRODUCCIÓN}

Desde la estandarización de la jerarquía de la evidencia planteada desde la perspectiva de la Medicina basada en evidencias (MBE), en la que se prepondera con justificada razón, al rol que cumplen los ensayos clínicos (EC) libres de sesgos, en la generación de evidencia que soporte las decisiones que de terapéutica se trate ${ }^{(1)}$, lo que sumado a la explosión de tecnología y desarrollo de nuevas moléculas para enfrentar las crecientes demandas de soluciones terapéuticas planteadas en las últimas décadas: cáncer, $\mathrm{VIH}$, obesidad, diabetes, hipertensión arterial, entre otras, han ubicado a los ensayos clínicos en un lugar preponderante de la investigación biomédica a nivel global, lo que se ve reflejado en la curva de progresión de la publicación de EC en la base de datos de PubMed, es así que, en el año 1995 se publicaron 21809 EC (2), número que ascendió a 36920 en el año 2011. Este incremento también se ha visto reflejado en la estadística de los estudios solicitados para aprobación y ejecución en el Perú, autorizando en ese periodo la realización de 1255 estudios (126 en el 2011) (3).

Además, debemos tener en consideración que los EC ejecutados en el Perú se han realizado en instituciones a todo nivel, hospitales de Essalud, del Ministerio de Salud y clínicas privadas ${ }^{(3)}$, de modo que se han extendido por todo tipo de instituciones dedicadas al cuidado de la salud.

Este panorama presentado sugeriría que el incremento y difusión en la ejecución de EC esté influyendo de manera positiva en la formación de una masa crítica que genere más evidencia que ayude a tomar decisiones en cuanto a problemas locales, lo cual intentaremos corroborar desde el punto de vista de temática; de formación de nuevos grupos de investigadores; de paralelismo en el incremento; de resolución de preguntas de investigación locales en temas álgidos; carga de enfermedad; endémicos y en plantas medicinales, e involucramiento de las instituciones nacionales en el patrocinio de ensayos clínicos en el Perú.

\section{TEMÁTICA DE LOS ENSAYOS CLÍNICOS EN EL PERÚ}

Para tener elementos de juicio se realizó una búsqueda exhaustiva en PubMed (buscador de los Institutos Nacional de Salud [NIH] de los EE. UU.), que permita tener una idea aproximada de la visibilidad internacional de ensayos clínicos en el Perú. Para ello, se hizo una búsqueda de términos MeSH con "ensayos clínicos" como "publication type" o "as a topic" cruzándolos con AND "Peru" OR "peruvian".
Sin restricciones de tiempo, se obtuvieron los siguientes resultados (2):

- Clinical trial[Publication type] AND Peru $=413$.

- Clinical trial[Publication type] AND peruvian= 113.

- [clinical trial as topic] AND Peru $=80$.

- [clinical trial as topic] AND peruvian $=10$.

Adicionalmente, se realizó una búsqueda en SCOPUS, para lo cual se utilizó la siguiente estrategia:

- (Clinical trial[Title] OR Clinical trial[Keyword] OR Clinical trial[Abstract]) AND Peru [AffilCountry] = 407.

- (Clinical trial[Title] OR Clinical trial[Keyword]) AND Peru [AffilCountry] $=377$.

- Excluyendo todas las filiaciones de los demás países: 66

Los abstract de los estudios obtenidos fueron revisados; aquellos estudios realizados en el Perú, que no formaban parte de ningún estudio multinacional y en los que se pudiera identificar a un grupo de investigación o investigador peruano, fueron identificados y se les denominó: "Ensayos clínicos aleatorizados de autoría peruana" (ECAP). Desde 1995, se identificó 102 de estos estudios.

De otro lado al revisar los estudios registrados en el Perú ante el Instituto Nacional de Salud, los estudios dirigidos a buscar terapias para enfermedades infectocontagiosas solo representaron el $15 \%$ del total de estudios ejecutados ${ }^{(3)}$; mientras que en los ECAP se han publicado en este periodo 55 estudios sobre enfermedades infectocontagiosas, es decir, más de la mitad, de los cuales 30 (casi un tercio del total) correspondieron al control de enfermedades parasitarias.

Al evaluar si existe un paralelismo entre el incremento sostenido en la solicitud y aprobación de ensayos clínicos patrocinados por la industria farmacéutica para su ejecución en el Perú (4), y su exponencial incremento, vemos que esto no ha ocurrido en las publicaciones peruanas sobre ensayos clínicos, ni en la variación a lo largo de estos años, en los que la producción de ECAP, se ha mantenido más o menos estable (Figura 1).

La evaluación de los 102 ECAP identicados permite, además, visualizar que durante este periodo, solo se han podido identificar en MEDLINE cinco estudios relacionados con la verificación de efectos beneficiosos de plantas medicinales ${ }^{(5-9)}$. Con relación a enfermedades endémicas, el ejemplo de enfermedad peruana por excelencia es "la enfermedad de Carrión", de la que no se ha hallado ningún ECA, información corroborada en 


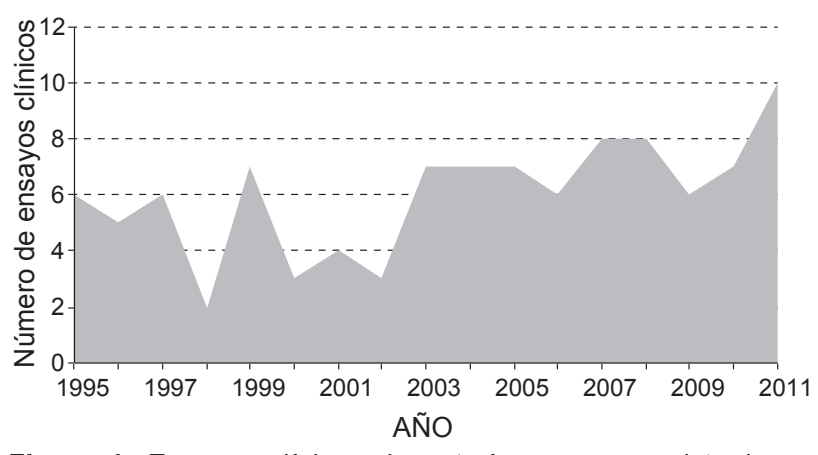

Figura 1. Ensayos clínicos de autoría peruana registrados desde 1995 a 2011.

una reciente revisión sistemática ${ }^{(10)}$.

\section{PATROCINIO DE ENSAYOS CLÍNICOS POR INSTITUCIONES LOCALES E INCORPORACIÓN DE NUEVOS GRUPOS DE INVESTIGACIÓN}

La pregunta es si el incremento en la ejecución de ensayos clínicos ha "contagiado" a instituciones peruanas para que estas se animen también a generar evidencia local. Los datos presentados por el Instituto Nacional de Salud (4), permiten apreciar con claridad que mientras hubieron $1170 \mathrm{EC}$ patrocinados por la industria farmacéutica internacional e instituciones de investigación y tecnología extranjeras, solo se han identificado cuatro EC patrocinados por asociaciones civiles nacionales o universidades peruanas. No hay registro en este periodo, de ensayos cínicos patrocinados por instituciones del estado.

Además, en la Figura 2 se aprecia que la producción de ECAP puede ser resumida a unos cuantos grupos,

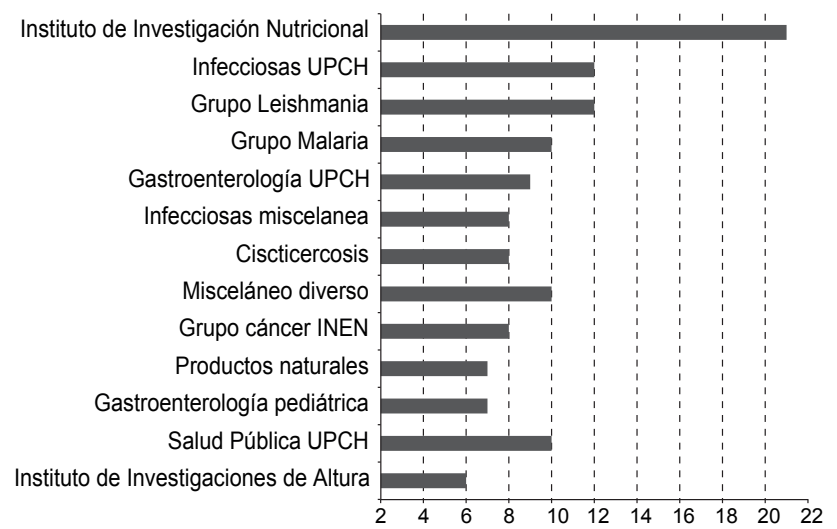

Figura 2. Número de ensayos clínicos autóctonos publicados desde 1995, por grupo de investigación.

UPCH: Universidad Peruana Cayetano Heredia; INEN: Instituto Nacional de Enfermedades Neoplásicas. los que se han mantenido en el tiempo $\mathrm{y}$, salvo la producción del grupo de cáncer del Instituto Nacional de Enfermedades Neoplásicas (INEN), el resto de grupos viene operando en el Perú desde hace más de una década

\section{ANÁLISIS PRELIMINAR}

La lectura de estos resultados preliminares nos deja varias sensaciones: la primera es que existe una discrepancia entre la ejecución de ensayos clínicos en general con relación a los que se producen de manera autóctona en el Perú, esto podría sugerir un desfase entre nuestras necesidades no resueltas y lo que se viene investigando, situación que se hace más sugestiva tras analizar la producción de ensayos clínicos autóctonos relacionados con nuestra carga de enfermedad (10), las que son lideradas en adultos por las enfermedades neuropsiquiátricas, tumores malignos y enfermedades cardiovasculares, pues no existiría ninguna relación con los ECAP producidos, y se hace urgente la redirección de los esfuerzos de investigación.

En relación con el punto anterior, este desfase también se pone de manifiesto cuando se evalúa la evidencia existente relacionada con nuestra enfermedad insigne, es decir, la enfermedad de Carrión, sobre la que no hemos sido capaces de generar ninguna evidencia en lo que a terapéutica se refiere y, de otro lado, la exigua producción y generación de evidencia en lo referente a la evaluación de los efectos benéficos de nuestra "riqueza biológica" tan ponderada coloquialmente a todo nivel, pero, sobre la que es necesaria la correspondiente verificación que permita su uso difundido, con los beneficios que esto acarrearía para los pueblos andinos y rurales de nuestro país de donde proviene la mayoría de estas plantas medicinales con supuestos efectos benéficos. Este punto ha sido antes abordado desde diversas perspectivas y la percepción siempre es la misma: Existe una pobre correlación entre la carga de enfermedad y los EC publicados en América Latina ${ }^{(11)}$, situación que no puede ser resuelta necesariamente con la realización de revisiones sistemáticas, las que tienen las mismas limitaciones que los EC; como tampoco pueden ser aplicables de manera directa por haber sido realizados en ambientes diferentes, contextos particulares y ajenos como son los países desarrollados ${ }^{(12)}$, situación que solo puede ser resuelta generando evidencia propia.

Al evaluar si el boom de ejecución de ensayos clínicos ${ }^{(4)}$ ha permitido el desarrollo de nuevos grupos de investigación, o nuevos investigadores, lo que se aprecia es que son los mismos grupos con presencia 
desde hace más de una década, muchos de ellos con líneas definidas de investigación, predominantemente dirigida a enfermedades infectocontagiosas y suplementos nutricionales; dichos grupos mantienen actividad en cuanto a publicaciones en revistas internacionales en las que abordan y buscan soluciones para problemas locales, en algunos casos con proyección internacional. Por lo tanto, en este punto se puede afirmar que a lo largo de estos años, el incremento de ensayos clínicos autorizados y ejecutados en el Perú no ha contribuido con el desarrollado de nuevos grupos de investigación.

Queda suficientemente clara la importancia del rol que cumplen las instituciones privadas extranjeras en el desarrollo y ejecución de ensayos clínicos en el Perú, pues, sin ellas, prácticamente no existirian ensayos clínicos en nuestro país, ya que no se ha logrado estimular a las instituciones académicas ni regulatorias peruanas a que se comprometan con la búsqueda y generación de evidencia para problemas locales, ni siquiera para estudios de fase IV; es decir, en los que se buscan nuevas aplicaciones para medicamentos ya existentes y adaptarlos a nuestra idiosincrasia ni estudios que intenten responder hipótesis o creencias relacionadas con propiedades medicinales para indicaciones especificas de diversos productos naturales.

Entonces, ante la pregunta de si contribuyen los ensayos clínicos con el desarrollo de la investigación en el Perú, vista como el abordaje de problemas locales, en búsqueda de soluciones a problemas de salud que aquejan a nuestra población y que se refleje en el desarrollo de nuevo conocimiento y nueva evidencia que ayude en la toma de decisiones relacionada con problemas de nuestros pacientes, en este momento, no se puede expresar una respuesta afirmativa.

De seguro se puede argüir en contra de todo lo mencionado, que en este mundo global la obtención de resultados sobre la prevención que provee una molécula nueva para el control de una enfermedad, debería hacerlo también en nuestro medio. Pongamos el ejemplo de la obtención de una nueva molécula para el control de la dislipidemia (un problema común) y su impacto en la prevención de eventos isquémicos cerebro-vasculares, e intentamos extrapolar y aplicar directamente el impacto de los resultados de la investigación realizada en pacientes de Europa o Norteamérica; sin embargo, si tenemos en cuenta en este punto que en nuestra población los niveles de LDL no son tan elevados y que el principal factor de riesgo es HDL bajo ${ }^{(13)}$, así mismo, en nuestro medio el tabaquismo no tiene el rol que tiene en esas regiones y que la proporción de ACV hemorrágicos es mayor que en la regiones mencionadas ${ }^{(14)}$, pues tendremos que es posible que no sea tan cierto, y que sea necesario que demandemos por evidencia regional con estudios que tengan validez externa sólida para nuestro medio y nuestros pacientes, en los que se evalúen los efectos beneficiosos/adversos para nuestra población.

Sabemos, sin embargo, que en general representa un reto llevar a cabo ensayos clínicos a iniciativa del investigador en países de bajos y medianos ingresos y lo discutido en cuanto a la pobre relación entre la investigación que se ejecuta y las necesidades locales no son particulares al Perú (15); y la realización de ensayos clínicos en ambientes de poblaciones vírgenes a tratamiento, enfermedades en estadios más avanzados, regulaciones éticas menos estrictas y menores costos operativos, siempre serán atractivos para empresas con fines de lucro ${ }^{(16)}$, de modo que nuestros entes rectores deben generar un equilibrio, favoreciendo la realización de ensayos clínicos a iniciativa de investigador local que responda ante necesidades de respuestas locales basados en la carga de enfermedad y endemia.

\section{BUSCANDO ALTERNATIVAS DE SOLUCIÓN}

Entonces, ¿qué podemos hacer para lograr que este incremento en la ejecución de ensayos cínicos en el Perú influya de manera directa y positiva en lo que a producción de ensayos clínicos que aborden problemas locales o regionales, que exploren y verifiquen si hallazgos en otras latitudes, son aplicables y tienen el mismo impacto que en nuestro medio?, creemos que la tarea no es fácil, pero empezar a discutir el tema seguro ayudará, para lo que sugerimos algunos puntos:

- Implementación de asociaciones universidad/ instituciones del estado (INS, DIGEMID) que actúen colaborativamente identificando áreas donde sea requerida la generación de evidencia.

- Revisar las barreras, si es que existen, para que instituciones sin fines de lucro, universidades e instituciones civiles puedan implementar y conducir ensayos clínicos.

- Diseñar una estrategia que permita la formulación y ejecución de ensayos clínicos teniendo en cuenta la carga de enfermedad en el Perú ${ }^{(10)}$, así como la evaluación de los potenciales efectos benéficos que tienen nuestras plantas medicinales y la solución a nuestros problemas endémicos de salud pública. 
- Destinar los fondos asignados a investigación por el Canon minero y otros actualmente subutilizados, para que, manejados por asociaciones público/privadas y con el soporte de las instituciones académicas, puedan ser utilizados en apoyo a iniciativas locales para idear, implementar y conducir ensayos clínicos, pago de seguros para pacientes y, en general, que permitan superar las barreras para la conducción de ensayos clínicos autóctonos en el Perú.

- Instituir desde las instancias regulatorias, la obligatoriedad de inversión de parte de los overhead que los ensayos clínicos producen en la formación de personal, no solo monitores, sino en la formación de científicos.

- Que se ponga énfasis desde las instituciones académicas no solo en la formación de investigadores epidemiológicos, sino que estos se capaciten para realizar intervenciones controladas en salud.

Es posible que las medidas planeadas no sean viables, o que se planteen más y mejores ideas que sí lo sean; sin embargo, lo más importante será que empecemos a discutir sobre el tema, que las instituciones privadas y estatales, entre las que deben estar las regulatorias como entes rectores, establezcan de manera conjunta las intervenciones y medidas pertinentes para revertir la situación mostrada e incentivar la formación de una masa crítica, con los recursos necesarios, sin trabas burocráticas de una legislación pensada en el patrocinio de la empresa privada necesariamente, y que respondan a las necesidades de evidencia en temas álgidos de nuestra realidad.

\section{CONCLUSIÓN}

A pesar de la proliferación de ensayos clínicos patrocinados por instituciones privadas con fines de lucro, se puede afirmar que estos no habrían influido en la generación de evidencia relacionada con la solución de problemas de terapéutica de nuestros problemas de carga de enfermedad, ni en problemas endémicos, no han influido en un aumento en la masa crítica de investigadores ni de nuevos grupos de investigadores que realicen ensayos clínicos en el Perú. No existe participación activa de instituciones estatales, universidades ni instituciones de la sociedad civil en la generación de evidencia a través del patrocinio de ensayos clínicos en el Perú.

Contribuciones de autoría: GM y AZR han participado en la concepción del artículo, la recolección de datos, su redacción y aprobación de la versión final.

Fuentes de financiamiento: autofinanciado.

Conflictos de interés: los autores declaran no tener conflictos de interés.

\section{REFERENCIAS BIBLIOGRÁFICAS}

1. Montori V, Devereaux PJ, Straus S, Haynes B, Guyatt G. Decision making and the patient. Advance topics in moving from evidence to action. In: User's guides to the medical literature: a manual for evidence based clinical practice. Second Edition. New York: Mc Graw Hill; 2008.

2. US National Library of Medicine, National Institute of Health [Internet]. Bethesda, MD: Pubmed; [citado el 2 de noviembre del 2012]. Disponible en: http://www.ncbi.nlm. nih.gov/pubmed/

3. Instituto Nacional de Salud. Estadística de los Ensayos Clínicos [Internet]. Lima: MINSA; [citado el 2 de noviembre del 2012]. Disponible en: http://www.ins.gob.pe/portal/ jerarquia/2/829/estadisticas-de-losensayos-clinicos/jer.829/
4. Minaya G, Fuentes D, Obregón C, Ayala-Quintanilla B, Yagui M. Características de los ensayos clínicos autorizados en el Perú, 1995-2012. Rev Peru Med Exp Salud Publica. 2012;29(4):431-6.

5. Garmendia F, Pando R, Ronceros G. Efecto del aceite de sacha inchi (plukenetia volúbilis 1) sobre el perfil lipídico en pacientes con hiperlipoproteinemia. Rev Peru Med Exp Salud Publica. 2011;28(4):62832.

6. Zegarra L, Vaisberg A, Loza C, Aguirre RL, Campos M, Fernandez I, et al. Double-blind randomized placebo-controlled study of Bixa orellana in patients with lower urinary tract symptoms associated to benign prostatic hyperplasia. Int Braz J Urol. 2007;33(4):493-500.
7. Gonzales GF, Córdova A, Vega K, Chung A, Villena A, Góñez C. Effect of Lepidium meyenii (Maca), a root with aphrodisiac and fertility-enhancing properties, on serum reproductive hormone levels in adult healthy men. J Endocrinol. 2003;176(1):163-8

8. Gonzales GF, Córdova A, Vega K, Chung A, Villena A, Góñez C, et al. Effect of Lepidium meyenii (MACA) on sexual desire and its absent relationship with serum testosterone levels in adult healthy men. Andrologia. 2002;34(6):367-72.

9. Sanchez Clemente N, Ugarte-Gil CA, Solórzano N, Maguiña C, Pachas P, Blazes D, et al. Bartonella bacilliformis: A Systematic Review of theLiterature to Guide the Research Agenda for Elimination. PLoS Negl Trop Dis. 2012;6(10):e1819. 
10. Perú, Ministerio de Salud. La carga de enfermedad y lesiones en el Perú. Lima: MINSA; 2008.

11. Perel P, Miranda JJ, Ortiz Z, Casas JP. Relation between the global burden of disease and randomized clinical trials conducted in Latin America published in the five leading medical journals. PLoS One. 2008;3(2):e1696.

12. Swingler GH, Volmink J, Ioannidis JP. Number of published systematic reviews and global burden of disease: database analysis. BMJ. 2003;327(7423):1083-4

13. Medina-Lezama J, Zea-Díaz H, MoreyVargas OL, Bolaños-Salazar JF, Muñoz-
Atahualpa E, Postigo-MacDowall M, et al. Prevalence of the MetabolicSyndrome in Peruvian Andean Hispanics: The PREVENCION study. Diabetes Res Clin Pract. 2007;78(2):270-81.

14. O’Donnell MJ, Xavier D, Liu L, Zhang H, Chin SL, Rao-Melacini P, et al. Risk factors for ischaemic and intracerebral haemorrhagic stroke in 22 countries (the INTERSTROKE study): a case-control study. Lancet. 2010;376(9735):112-23.

15. Mbuagbaw L, Thabane L, OngoloZogo P, Lang T. The challenges and opportunities of conducting a clinical trial in a low resource setting: the case of the Cameroon mobile phone SMS (CAMPS) trial, an investigator initiated trial. Trials. 2011;12:145.

16. Devasenapathy N, Singh K, Prabhakaran D. Conduct of clinical trials in developing countries: a perspective. Curr Opin Cardiol. 2009; 24(4):295-300.

Correspondencia: Germán Málaga Rodríguez Dirección: Calle Chavin 159, CC Monterrico. Santiago de Surco. Lima 33. Perú

Teléfono: (51) 992768300

Correo electrónico: german.malaga@upch.pe

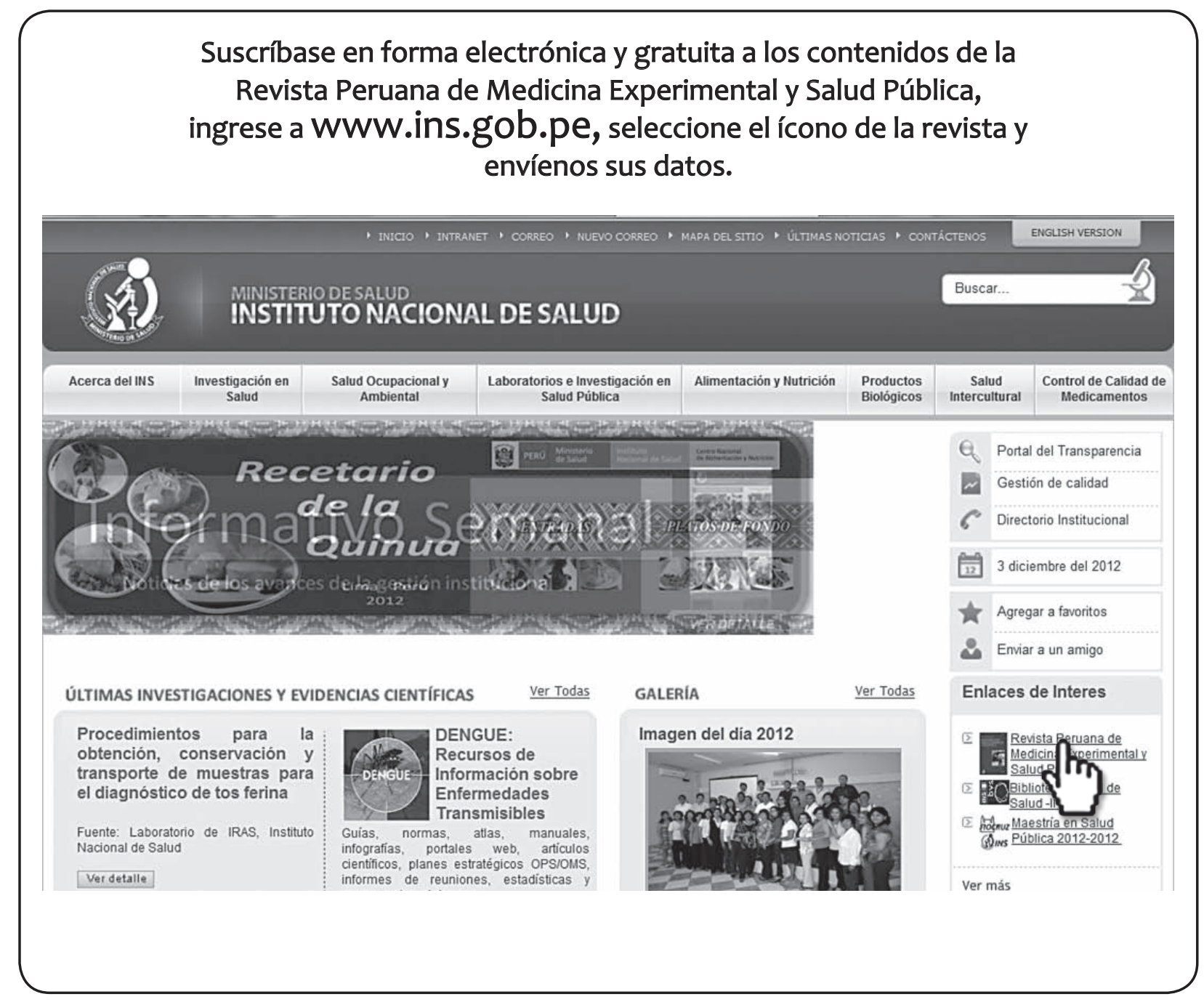

